\title{
Research
}

\section{Canadian paramedic experience with intramuscular ketamine for extreme agitation: A quality improvement initiative}

Jennie Helmer BCom, MEd is a Paramedic Practice Leader ${ }^{1}$ and PhD Student'; joe Acker ACP, MA is Director of Clinical and Professional Practice ${ }^{1}$, Adjunct Senior Lecturer ${ }^{3}$ and Adjunct Professor ${ }^{2}$; Jon Deakin is a Paramedic Practice Leader ${ }^{1}$ and Lead Educator'; Tania Johnston ACP, BScN, MHS is a Lecturer in Paramedicine ${ }^{3}$

\author{
Affiliations: \\ 'British Columbia Emergency Health Services, Vancouver, Canada \\ 2University of British Columbia, Faculty of Medicine, Vancouver, Canada \\ ${ }^{3}$ Charles Sturt University, School of Biomedical Sciences, Port Macquarie, New South Wales \\ ${ }^{4}$ The Justice Institute of British Columbia, Vancouver, Canada
}

https://doi.org/10.33151/ajp.17.763

\section{Abstract}

\section{Background}

There are no published reports in Canada examining paramedic use of ketamine for highly agitated patients or excited delirium syndrome. We employed a Plan, Do, Study, Act (PDSA) quality improvement approach to evaluate the safety and effectiveness of advanced care paramedic administered intramuscular (IM) ketamine for patients with extreme agitation in the out-of-hospital setting.

\section{Methods}

Data were prospectively collected from July 2018 to January 2019 when advanced care paramedics with specific training administered IM ketamine as an alternative to midazolam. Paramedics used a clinical audit form to document the ketamine dose, patient response on the Richmond Agitation Sedation Scale (RASS) at time intervals, adverse effects, and any airway management interventions they performed.

\section{Results}

Thirty-three patients received either $4 \mathrm{mg} / \mathrm{kg}$ or $5 \mathrm{mg} / \mathrm{kg}$ of ketamine. Combining data for both doses, the median change in RASS score at 5 minutes post-ketamine was 3 (range 0 to 8 ) and statistically significant for each dose. There were seven cases (21\%) with reported adverse effects including SpO2 <90\% (3/7), hypersalivation (3/7), trismus or teeth grinding (2/7), muscular rigidity (1/7) and laryngospasm (1/7). Statistical analysis confirmed that the incidence of adverse events was not dose dependent. Basic airway management was performed in one-third of all cases.

\section{Conclusion}

We piloted the implementation of ketamine for sedation in our paramedic system by employing a PDSA cycle. Ketamine $5 \mathrm{mg} / \mathrm{kg}$ IM provided effective control of acutely agitated patients with adequate sedation at 5 minutes post-delivery. Any adverse events that occurred as a result of IM ketamine were readily managed with basic airway management interventions.

Keywords:

ketamine; sedation; paramedic; PDSA; agitation; excited delirium; emergency medical services 


\section{Introduction}

Paramedics are routinely called to manage highly agitated and combative patients. A subset of these patients are suffering from excited delirium syndrome (ExDS), an acute behavioural disorder coupled with metabolic disturbances that may be fatal if not treated in a timely manner (1). Though the pathway to death in ExDS is unknown, experts suggest it will progress if left untreated, causing a combination of acidosis, sympathomimetic surge and hyperthermia. The estimated mortality rate for ExDS ranges from $8.3 \%$ to $16.5 \%$ (2-5). Patients experiencing ExDS are more likely to have an underlying mental health condition and to use illicit drugs (5). Ultimately, they present as a danger to themselves as well as to paramedics and other emergency responders.

Paramedicine is practised in uncontrolled environments (6) where practitioners are exposed to a relatively high risk of occupational injury (7). This is compounded when paramedics encounter highly agitated patients whose combative behaviours can cause significant injury or harm. This risk is extended to police officers and fire fighters who are often called to assist with managing combative patients. In extreme circumstances, British Columbia Emergency Health Service (BCEHS) paramedics may use physical restraints on aggressive patients to safely transport them to the emergency department (8).

As an alternate strategy, a subset of BCEHS paramedics can employ sedation as a pharmacological intervention. Advanced Care Paramedics (ACPs) in our system have traditionally administered a benzodiazepine when standard de-escalation proves ineffective. Our clinical practice guideline for managing highly agitated patients includes midazolam $5-10 \mathrm{mg}$ via intramuscular (IM) injection with an option to repeat the dose once if needed (8). IM midazolam has drawbacks when patients require rapid and safe sedation (9) including an onset time of 15-20 minutes and an associated risk of cardiorespiratory compromise (10). To manage extreme agitation in the emergency department, physicians are more likely to use benzodiazepines as an adjunct to antipsychotics such as haloperidol or droperidol (11-14). Recently, ketamine has been introduced to the out-of-hospital setting as a feasible alternative for the purpose of sedation.

Paramedics are now using ketamine, a dissociative anaesthetic, for a range of indications (15-18). The effects of ketamine are known to be dose dependent. Its effect on cognition is attributed to antagonism at the $\mathrm{N}$-methyl-d-aspartate (NMDA) receptor; effectively disrupting communication pathways in the brain (19). Previous Canadian studies include the administration of high dose ketamine as an adjunct for endotracheal intubation in the air ambulance environment (18) and in smaller doses as an analgesic in the ground ambulance setting $(20,21)$. In a recent survey of 10,737 American paramedics, 33\% reported giving varying doses of ketamine for analgesia, intubation and sedation (22). Patients who receive high doses of ketamine enter a cataplectic state. They maintain their airway reflexes and cardiorespiratory function but cannot perceive external stimuli or interact in any way (5). This makes it a useful option to manage extreme agitation, and paramedic services in the United States and Australia are now widely using ketamine for this purpose $(4,16,17,23-27)$. American researchers report observing varying incidence rates of adverse effects secondary to ketamine including laryngospasm and hypersalivation $(2,23)$. There have also been reports of high intubation rates when ketamine was given pre-hospital $(2,17,28)$. To the best of our knowledge, there are no published studies of a Canadian paramedic service using ketamine for extreme agitation.

On reviewing the published literature and safety profile of ketamine in 2018, our clinical governance committee initially approved our advanced care paramedics to use ketamine for analgesia and endotracheal intubation. Subsequently, the committee approved a trial to study the use of ketamine as an alternative to midazolam for sedation. We employed a PDSA quality improvement approach to evaluate the safety and effectiveness of advanced care paramedic administered IM ketamine for patients with extreme agitation in our prehospital setting. We determined safety based on paramedic reported incidence of adverse effects and airway intervention. Effectiveness was evidenced by a change in the Richmond Agitation Scale Score (RASS) from the patient's original to a target of +2 to -2 at 5 minutes post ketamine administration.

\section{Methods}

\section{Study design and setting}

We performed this quality improvement study by using a PDSA cycle. Speroff and O'Connor describe how a four-step PDSA project aims to determine whether a suggested change is actually an improvement.

1. Plan - predict what will happen and plan for data collection.

2. Do - carry out the plan using a study protocol and collect the data.

3. Study - analyse, interpret and summarise the results.

4. Act - determine any changes required and implement the improvement initiative (29).

Serving a provincial population of $4,810,000$, BCEHS responds to more than 750,000 calls annually (30). Of these events in 2018 , approximately 3400 patients were identified as potentially experiencing severe agitation; and 120 agitated patients were pharmacologically sedated with IM midazolam. The option to give sedation is limited to our 360 advanced and critical care paramedics who account for approximately $12 \%$ of our paramedic workforce. The majority of severely agitated patients are treated and transported solely by primary care paramedics who are not licensed to administer sedation.

In 2018, our paramedic service decided to trial whether the paramedics could use ketamine as an alternative to midazolam 
for sedation. This was in response to ACPs documenting concerns over an increased incidence of encounters with agitated patients and their perception that midazolam was an inadequate sedative. ACPs use the RASS to measure and document patient sedation. They reported that patients were requiring repeated doses of midazolam to achieve a low enough RASS score for safe transport, posing an increased risk to patient and practitioner safety.

The RASS is a validated observational instrument used to assess levels of patient sedation and agitation. It uses a 10-point scale (+4 to -5) with discrete criteria for levels of sedation and agitation (31). Using the RASS tool, we aimed to measure the effectiveness of advanced care paramedic administered IM ketamine to reduce patient agitation.

Consistent with our clinical practice guidelines, we assessed the effectiveness of sedation by comparing the patients' original RASS score against a target of +2 to -2 at 5 minutes after drug administration. We selected a 5-minute interval as a practical timeframe for paramedics to rapidly gain control of volatile situations. We also asked paramedics to report their levels of satisfaction as an additional endpoint for effectiveness. To measure the second objective of patient safety, ACPs documented adverse effects that occurred post ketamine administration and anytime they performed basic or advanced airway management procedures.

Based on a review of contemporary literature and emergency physician expert advice, our prediction was that a single dose of $4 \mathrm{mg} / \mathrm{kg}$ IM ketamine would be a viable alternative to midazolam and achieve adequate sedation levels without compromising patient safety $(15,16)$. Our PDSA project plan involved allowing a select group of ACPs in two major metro centres to use ketamine as a first line sedative for patients with extreme agitation. In total, 36 paramedics from two centrally located ambulance stations were trained and eligible to take part in the project.

The ACPs received approximately 2 hours of education on the pathophysiology and accurate recognition of severe agitation and ExDS as well as the pharmacodynamics and administration of ketamine. We also provided a refresher on the use of RASS (Table 1). The ACPs completed an online learning module followed by an in-person session led by the primary investigators. Paramedics were required to pass an on-line knowledge assessment and successfully complete a case simulation during the face-to-face session. The paramedics concurrently completed a two-day Advanced Airway Interventions and Management in Emergencies (AIME) course before being able to administer ketamine (32). In addition, all receiving hospitals were notified of the new protocol.

Consistent with our organisation's policy, we assessed the project using our ethics guidelines screening tool. As a quality assurance study that was used for standard program activities, our project was deemed to have minimal risk implications and was exempt from institutional board review.

Table 1. The Richmond Agitation Sedation Scale

\begin{tabular}{|c|c|c|}
\hline Score & Term & Description \\
\hline+4 & Combative & $\begin{array}{l}\text { Overtly combative or violent; } \\
\text { immediate danger to staff }\end{array}$ \\
\hline+3 & Very agitation & $\begin{array}{l}\text { Pulls on or removes tube(s) or } \\
\text { catheter(s) or has aggressive } \\
\text { behaviour toward staff }\end{array}$ \\
\hline+2 & Agitated & $\begin{array}{l}\text { Frequent non-purposeful movement } \\
\text { or patient-ventilator dyssynchrony }\end{array}$ \\
\hline+1 & Restless & $\begin{array}{l}\text { Anxious or apprehensive but } \\
\text { movements not aggressive or } \\
\text { vigorous }\end{array}$ \\
\hline 0 & Alert and calm & \\
\hline-1 & Drowsy & $\begin{array}{l}\text { Not fully alert, but has sustained } \\
\text { (more than } 10 \text { seconds) awakening, } \\
\text { with eye contact, to voice }\end{array}$ \\
\hline-2 & Light sedation & $\begin{array}{l}\text { Briefly (less than } 10 \text { seconds) } \\
\text { awakens with eye contact to voice }\end{array}$ \\
\hline-3 & $\begin{array}{l}\text { Moderate } \\
\text { sedation }\end{array}$ & $\begin{array}{l}\text { Any movement (but no eye contact) } \\
\text { to voice }\end{array}$ \\
\hline-4 & Deep sedation & $\begin{array}{l}\text { No response to voice, but any } \\
\text { movement to physical stimulation }\end{array}$ \\
\hline-5 & Unarousable & $\begin{array}{l}\text { No response to voice or physical } \\
\text { stimulation }\end{array}$ \\
\hline
\end{tabular}

Source: Sessler CN, Gosnell M, Grap MJ, et al (2002)

\section{Participants}

Patients meeting inclusion criteria were defined as having severe agitation and combativeness consistent with acute behavioural disturbance due to potential ExDS with a RASS score of +4 (combative) to +3 (very agitated) who could not be safely conveyed to the hospital without pharmacological intervention. Patients of all ages who fit this criterion and were given ketamine by a study-trained paramedic between July 2018 and January 2019 were included in the study. Patients who required sedation for RASS scores less than +3 received midazolam per the standard treatment guidelines and were not included in the project.

\section{Protocol}

The following project protocol describes the actions representing 'do' in our PDSA cycle. All patients who met the inclusion criteria initially received $4 \mathrm{mg} / \mathrm{kg}$ IM ketamine. In the first 3 months of the study, paramedics signalled a safety concern by submitting incident reports of insufficient patient sedation. The clinical governance committee reviewed these incidents and in October 2018 we subsequently decided to increase the ketamine dose to $5 \mathrm{mg} / \mathrm{kg}$ which is the upper limit reported in published literature $(4,17)$. 
Paramedics estimated the patient's weight in most cases. Using a purpose-built electronic clinical audit form, the ACPs documented each time they gave ketamine for extreme agitation and signs of ExDS. The completed form was submitted directly to a primary investigator. This triggered an alert to the medical director who then contacted each paramedic crew by telephone as soon as possible to discuss the case, especially if any adverse effects were observed.

In addition to the patient's age and gender, the pre- and postketamine RASS were recorded at time 0,5 and 10 minutes and on arrival to the emergency department. Paramedic perception of sedation effectiveness was measured using a Likert scale of 1 (no effect) to 5 (very effective). They also documented the total time to adequate sedation as a record of 'administration-to-sedation duration' in minutes. Using a dropdown menu, paramedics recorded the use of airway adjuncts including jaw thrust, nasopharyngeal airway, oropharyngeal airway, supraglottic airway and endotracheal intubation, as well any adverse effects including vomiting, $\mathrm{SpO} 2<90 \%$, hypersecretions requiring suctioning, trismus, muscle rigidity and laryngospasm. Appreciating that the aforementioned adverse effects may also be symptoms associated with the patient's primary complaint, we advised paramedics to use their best judgement to determine if they occurred as a result of ketamine administration. Paramedics rated the adequacy of the duration of sedation and the suitability of training materials using binary yes or no responses on the data collection form and had a free text form for additional comments. We were able to document the initial dispatch assigned event code for each case. Paramedics also recorded standard observations such as the patient's Glasgow Coma Score, temperature, blood pressure and heart rate on the patient care record but these are not reported as part of this project.

\section{Data analysis}

Representing the 'study' step in our PDSA cycle, our data analysis and results are presented next. The data were collected using an electronic form and manually collated in an Excel $^{\mathrm{TM}}$ spreadsheet (Microsoft Corporation). Frequencies and descriptive statistics were analysed using IBM SPSS Version 25. To answer the question of effectiveness, our primary outcome was a change in RASS scores to a target range between +2 and -2 at 5 minutes after ketamine administration. In addition, we used paramedic reports of time to adequate sedation and their perceptions of effectiveness as secondary measures. Outcomes related to the safety of ketamine administration included quantifying the type and frequency of adverse effects observed as well as type and frequency of airway adjuncts that paramedics used.

\section{Results}

\section{Sample characteristics}

A total of 34 patients received ketamine sedation for extreme agitation during the project period. One patient was excluded when it was determined that the patient had a traumatic brain injury rather than an acute behavioural disturbance. Analysis of aggregate data reveals that of the 33 remaining patients included in the project, $61 \%$ were male with an average age of 30 years. Within the study population, 19/33 $(58 \%)$ patients were identified as having ingested illicit drugs including one or more of cocaine, lysergic acid diethylamide, methamphetamines, heroin, marijuana, ketamine and opioids. This was documented as 'none' for one patient and 'unknown' in 13 cases. The characteristics of the patients who received ketamine are summarised in Table 2 . The primary dispatch assigned event codes were for psychiatric/abnormal behaviour $(42 \%)$ and overdose/poisoning (18\%). The additional codes are presented in Table 3.

Table 2. Characteristics of cases with patients who received ketamine $(n=33)$

\begin{tabular}{|ll|}
\hline Characteristic & \\
\hline Age: mean (SD), (range 17-56 years) & 30.4 \\
& $(11.2)$ \\
Gender: male, \% & $61 \%$ \\
Dispatch code: psychiatric/abnormal behaviour, \% & $42 \%$ \\
Accompanying intoxicant documented: \% & $58 \%$ \\
\hline
\end{tabular}

Table 3. Dispatch-assigned event codes

\begin{tabular}{|l|c|}
\hline Dispatch code & \# Cases \\
\hline psychiatric/abnormal behaviour & 14 \\
\hline overdose/poisoning & 6 \\
\hline unconscious & 2 \\
\hline assault & 2 \\
\hline sick person & 2 \\
\hline seizure & 2 \\
\hline burns & 1 \\
\hline animal bite & 1 \\
\hline heart problem & 1 \\
\hline interfacility transfer & 1 \\
\hline haemorrhage & 1 \\
\hline
\end{tabular}

\section{Ketamine administration and time to adequate sedation}

Throughout the project, $42 \%(\mathrm{n}=14)$ received $4 \mathrm{mg} / \mathrm{kg}$ of IM ketamine and $58 \%(\mathrm{n}=19)$ received $5 \mathrm{mg} / \mathrm{kg}$. The mean IM total dose of ketamine was $379 \mathrm{mg}$ (range 150-600 mg, SD $113 \mathrm{mg}$ ); 10 cases did not have the time to adequate sedation entered. In 23/33 of complete records, the time to adequate sedation was reported as a mean of 4.7 minutes (range $0.5-13$ minutes, SD 3.5 minutes).

\section{RASS scores and reported effects}

All patients entered in the study had an initial RASS of either $+4(n=20)$ or $+3(n=13)$. We are reporting the median scores as RASS score data were unevenly distributed. 
Though it was not part of the original study protocol, we are also reporting our RASS findings based on the administered dose (Table 4).

Mann-Whitney testing on the RASS score at 5 minutes (median 0 , range $0-8$ ) revealed an equivalent reduction for patients with an initial RASS of +4 or $+3(p=0.102)$. When combining data on both doses, the overall median change in RASS score at 5 minutes post-ketamine administration was 3 (range 0 to 8). The change in RASS at 5 minutes was determined to be statistically significant for all patients who received $4 \mathrm{mg}(\mathrm{p}=0.0002)$ or $5 \mathrm{mg}(\mathrm{p}<0.0001)$ (Figure 1). When comparing doses however; our data did not demonstrate a statistically significant difference in the change in RASS at 5 minutes post-ketamine $(p=0.494)$.

Of interest is that $57 \%$ (8/14) of patients who received $4 \mathrm{mg} /$ $\mathrm{kg}$ had a change in RASS score of $<4$ after 5 minutes and that on arrival to the emergency department, $45 \%(n=13)$ of all patients had a recorded RASS score of -4 (deep sedation) or -5 (unarousable).

The paramedics reported that 'effective' or 'very effective' sedation was achieved in $82 \%$ (27/33) of patients. In the four cases in which paramedics reported dissatisfaction with the sedation, the patients remained with a RASS of +3 at 10 minutes post-ketamine administration. Three of these patients had been given $5 \mathrm{mg} / \mathrm{kg}$ of IM ketamine and had reportedly used one or more of methamphetamines, ketamine, heroin or other opioids.

\section{Adverse effects and airway interventions}

Focussing on the primary endpoint to measure patient safety, there were seven cases $(21 \%)$ with reported adverse effects including SpO2 $<90 \%(3 / 7)$, hypersalivation (3/7), trismus or teeth grinding (2/7), muscular rigidity (1/7) and laryngospasm (1/7) (Table 5). In the one reported incidence of laryngospasm, the paramedics discussed the case with the medical director and concluded that this was the most likely cause of the patient's presentation. It was managed with short-term bag valve mask ventilation. Though the majority $(n=5)$ of cases with recorded adverse events occurred at the higher dosage range of $5 \mathrm{mg} / \mathrm{kg}$, statistical analysis confirmed that the incidence of adverse events was not dose dependent ( $p=0.67$, Fishers exact test).

Airway management data revealed that $11 / 33(33 \%)$ patients received some form of basic airway management and none required a supraglottic airway or endotracheal intubation. $14 \%$ (2/14) of patients who received $4 \mathrm{mg} / \mathrm{kg}$ of ketamine

Table 4. Median and range RASS scores over time (by dose of ketamine)

\begin{tabular}{|llllll|}
\hline & & Initial RASS & 5 minutes & 10 minutes & ED arrival \\
\hline Dose & $\mathrm{n}$ & & & & \\
$4 \mathrm{mg} / \mathrm{kg}$ & 14 & $+4($ range +4 to +3$)$ & $+1($ range +3 to -3$)$ & $-2($ range +3 to -4$)$ & $-2($ range +3 to -4$)$ \\
$5 \mathrm{mg} / \mathrm{kg}$ & 19 & $+4($ range +4 to +3$)$ & $0($ range +4 to -4$)$ & $-3($ range +3 to -5$)$ & $-4($ range +4 to -5$)$ \\
\hline
\end{tabular}
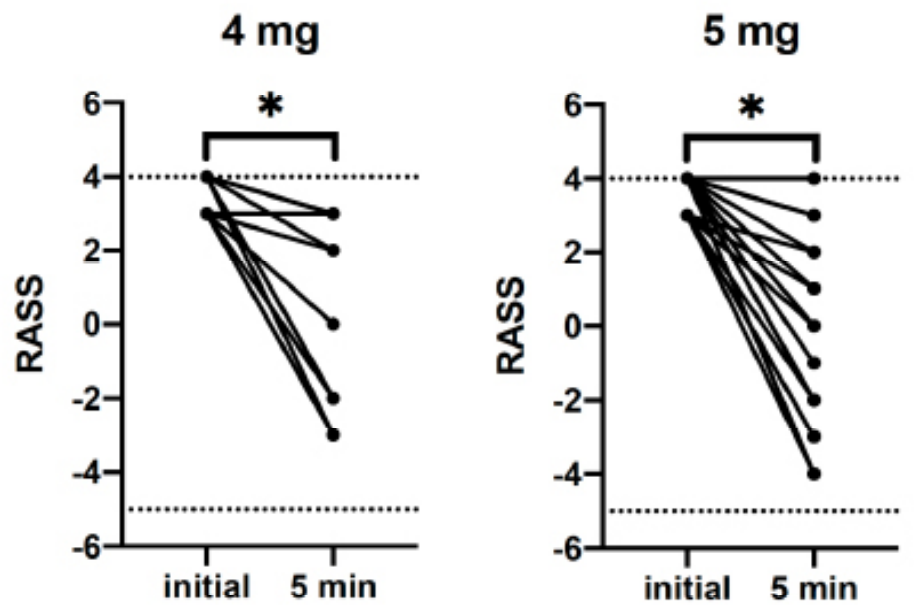

Figure 1. Ranked change in RASS scores at 5 minutes by dose 
required placement of either an oropharyngeal airway (OPA) or nasopharyngeal airway (NPA), while 47\% (9/19) who received $5 \mathrm{mg} / \mathrm{kg}$ required either a jaw thrust (1/19), basic airway adjunct in the form of an OPA or NPA (7/19) or ventilatory support with a bag valve mask (BVM) (1/19). In eight cases, paramedics performed a basic airway intervention but did not report an adverse event. When comparing the two doses of ketamine, the differences in airway management was not found to be statistically significant ( $p=0.067$, Fishers exact test).

\section{Discussion}

Our aim was to determine the safety and effectiveness of paramedic administered IM ketamine for highly agitated patients in the out-of-hospital setting. As part of the 'act' step in our PDSA project, we offer the following discussion points for consideration. With respect to effectiveness, our paramedics reported that they were satisfied that ketamine achieved favourable results in the majority of situations. We quantified this with RASS scores describing most patients presenting as either 'restless' or 'alert/calm' 5 minutes after ketamine was given. Of the 23/33 cases where it was recorded, our findings demonstrate that effective sedation occurred in a mean of 4.7 minutes. This is consistent with Cole et al who found that ketamine was effective in 5 minutes as compared to haloperidol in 17 minutes (2). Similarly, Riddell et al found ketamine to have a faster onset than benzodiazepines in their study of 98 emergency department patients (11). In their systematic review of ketamine in the emergency department and out-of-hospital settings, Mankowitz et al report an average 7.2 minutes to adequate sedation (23) while Scheppke et al achieved effective sedation and medical control in just over 2 minutes (16). Overall the research shows varied results when looking at time to adequate sedation as a measure of effectiveness.

During the course of our project we used a dose of $4-5 \mathrm{mg} /$ $\mathrm{kg}$ which is within the parameters of high dose ketamine for sedation described in pre-hospital literature. Horton and Scheppke et al each used $4 \mathrm{mg} / \mathrm{kg}$ in their respective studies of 52 patients while Olives et al reports using $5 \mathrm{mg} / \mathrm{kg}$ in a study involving 135 cases (15-17). Similarly, seven patients in a study by Scaggs et al were also given $5 \mathrm{mg} / \mathrm{kg}$ (4). Our paramedics determined the lower dose to be inadequate despite no statistically significant difference in the change of a patient's RASS score at 5 minutes when comparing doses.
The mean ketamine dose of $379 \mathrm{mg}$ we recorded is higher than the $324 \mathrm{mg}$ reported by Keseg et al and Mankowitz et al whose review of 650 patients across 18 pre-hospital and emergency studies revealed an average IM dose of $315 \mathrm{mg}(23,28)$. Of interest is that we did not specify a maximum dose in our study. In their literature review of pre-hospital ketamine for ExDS and agitation, Linder et al also noted that paramedics did not typically have a maximum dose and recommends that one should be set (19).

Our paramedics also recorded an estimated weight on only $60 \%$ of patients which is comparable to a study by Hollis where paramedics recorded weights on 26/38 patients (25). Research on paramedic weight estimation is mixed (33-35) and we are unable to confirm whether the ACPs estimated weights with any degree of accuracy, something that would affect the dose of ketamine given, and would require further study.

We reviewed our findings on the effectiveness of ketamine in the context of Campeau's Space-Control Theory of Paramedic Scene Management that highlights how hazardous and complex the out-of-hospital environment can be. Campeau emphasises the important role that paramedics have in bringing order to chaotic scenes and how they have to find a balance between providing care while ensuring safety for both the patient and practitioners (6). In this project, we used the RASS as a quantitative measure to evaluate how effective ketamine is when given for extreme agitation - that is, did it achieve the desired result? While our findings demonstrate that RASS scores will decrease with ketamine, they are unable to quantify other more complex aspects of effectiveness. We recognise that these findings must be interpreted within a broader context and that effectiveness, in the opinion of a paramedic at the scene, extends beyond a RASS score. For example, a patient with a high potential for a communicable disease that has been spitting at emergency responders may still pose a potential threat despite a reduced RASS score. In addition, the number and type of resources at a scene who can assist during the transport of a patient with a known history of violence may impact whether a paramedic feels that a patient is adequately sedated.

In our experience, most patients with acute behavioural disorder showed a significant reduction in agitation with ketamine, thus reducing the risk of ExDS and its potential sequelae. We also

Table 5. Incidences of adverse effects (by dose of ketamine)

\begin{tabular}{|llllll|}
\hline Adverse effect & SpO2 $<\mathbf{9 0 \%}$ & Hypersalivation & $\begin{array}{l}\text { Trismus/teeth } \\
\text { grinding }\end{array}$ & Muscular rigidity & Laryngospasm \\
\hline $4 \mathrm{mg} / \mathrm{kg}$ & 1 & 1 & & & 1 \\
$5 \mathrm{mg} / \mathrm{kg}^{*}$ & 2 & 2 & 2 & 1 & 1 \\
Total & 3 & 3 & 2 & & 1 \\
\hline
\end{tabular}

${ }^{*}$ Two patients had more than one adverse event recorded 
examined patient safety by measuring adverse effects attributed to ketamine, especially those that required subsequent airway management. Our findings include adverse effects reported in $7 / 33$ cases (21\%) including SpO2 <90\% $(9 \%)$, hypersalivation $(9 \%)$, trismus $(7 \%)$, muscle rigidity $(3 \%)$ and laryngospasm (3\%). This experience with ketamine differs from Mankowitz et al who found a documented average hypoxia rate of $1.8 \%$ and $18.8 \%$ hypersalivation post-ketamine. They do report a comparable rate of laryngospasm at 1.3\% (23). Notably, Cole et al report hypersalivation in $38 \%$ of cases and laryngospasm in $5 \%$ of patients $(n=64)(2)$. We are unable to explain the low incidence of observed hypersalivation in our patient population. Though not included in our project, the research also identifies emergence reactions as a commonly reported adverse effect of ketamine $(19,23,36)$.

To manage adverse effects, $30 \%$ (10/33) of our patients received basic airway management in the form of a jaw thrust, placement of an airway adjunct (OPA or NPA) and, in one case, bag-valve mask ventilation. In our experience, the majority of adverse events and subsequent airway interventions occurred with the higher dose of $5 \mathrm{mg} / \mathrm{kg}$, though this was not a statistically significant finding. Follow-up telephone calls by the medical director to the paramedics did not reveal any additional concerns beyond those reported on the audit forms.

It is noteworthy that none of the patients in our project required an advanced airway in the out-of-hospital environment. This contradicts a recurring theme in the ketamine literature that associates its use in agitation with high rates of intubation. Examples of intubation rates for agitated patients receiving pre-hospital ketamine are reported by American researchers Olives et al (17) at 63\% (85/135), Cole et al (2) at 39\% (25/64), and by Keseg et al (28) at $22 \%(8 / 35)$. As we did not follow our patients beyond the pre-hospital setting, we are unable to report on the incidence of intubation in the emergency department. Given that none of our ACPs elected to intubate, which is within their scope of practice, we interpret this as a surrogate finding that our patients did not require advanced airway management. This may be due to the paramedic comfort level with patients who are sedated yet stable and still maintaining their airway with minimal intervention. Olives et al suggest that the high rates of intubation in their study may be due to a lack of provider comfort when dealing with sedated patients post-ketamine administration (17). This explanation is further supported by Cole et al who suggest in a follow-up report that some of the intubations reported in earlier studies may have been avoidable and involved practitioners misapplying the GCS axiom 'GCS less than 8 , intubate' (1). Cole recently coined the term 'GCS-3K' to explain the phenomenon that although patients given high doses of ketamine are a GCS-3, it is due to the ketamine alone and deliberate dissociative anaesthesia. These patients are not obtunded as a result of shock, respiratory failure, metabolic derangement or head injury and therefore the conventional teaching around airway management may be misleading (2).

\section{PDSA outcome}

Following the PDSA methodology of this quality improvement project, we proceeded to 'act' on our findings. Our experience enabled us to implement ketamine for use by all ACPs in our paramedic system to manage severe agitation and potential ExDS. On reviewing the data, we updated the clinical practice guidelines for ACPs in October 2019 to include ketamine 5 $\mathrm{mg} / \mathrm{kg} \mathrm{IM}$ as a first line option for severe agitation (RASS +4 to +3 ). We recognise that approximately $33 \%$ of patients who receive high dose ketamine may require some form of airway intervention. For this reason, all ACPs will continue to receive initial ketamine training in conjunction with the AIME course. During AIME they are taught a staged approach to airway management including basic, intermediate and advanced airway skills to ensure they have the knowledge, skills and ability to anticipate and manage any airway compromise or adverse effects following the administration of ketamine.

In summary, after studying its effectiveness and safety using a PDSA cycle, ketamine $5 \mathrm{mg} / \mathrm{kg}$ IM has now been implemented for ACP use for undifferentiated patients experiencing extreme agitation and potential ExDS. We continue to monitor this strategy through our regular quality improvement audits. The lessons learnt through this project also include the importance of comprehensive data collection and analysis for new clinical initiatives as well as the need for paramedics to provide high-quality patient documentation and complete quality improvement surveys to help monitor and advance clinical practice.

\section{Limitations}

This project has several important limitations. First, we relied on paramedics to complete the clinical audit form after the patient encounter which may introduce bias into the data collection. Second, we used paramedic-assessed RASS scores, which have been shown to differ from scores calculated in the emergency department (36). We also determined that RASS scores alone may not comprehensively measure sedation in the out-of-hospital setting, and that another objective tool would be useful. Third, this study has a small sample size and the results of this sample cannot be used to generalise a larger patient population. In addition, our study did not extend beyond the pre-hospital setting, so we are unable to report on any additional adverse effects, medical interventions, including in-hospital advanced airway management or patient outcomes. Further research is needed to better understand effectiveness and safety of ketamine when managing highly agitated patients in the unique context of the paramedic environment. While this quality improvement project adds to the body of knowledge, more high-quality research needs to be conducted to assess the role of ketamine for extreme agitation and ExDS in the prehospital setting. 


\section{Conclusion}

By employing a PDSA cycle, we were able to pilot the implementation of ketamine for treatment of acute agitation with sedation in our paramedic system. In our experience, ketamine administered at $5 \mathrm{mg} / \mathrm{kg} \mathrm{IM}$ provided effective sedation 5 minutes post-delivery to highly agitated and combative patients. Any adverse events that occurred as a result of IM ketamine were readily managed with basic airway management interventions.

\section{Competing interests}

The authors declare no competing interests. Each author of this paper has completed the ICMJE conflict of interest statement.

\section{References}

1. Cole J, Driver B, Klein L, et al. In reply: Ketamine is an important therapy for prehospital agitation. Its exact role and side effect profile are still undefined. Am J Emerg Med 2018:36:502-3.

2. Cole JB, Moore JC, Nystrom PC, et al. A prospective study of ketamine versus haloperidol for severe prehospital agitation. Clin Toxicol 2016;54:556-62.

3. Gonin P, Beysard N, Yersin B, Carron PN. Excited delirium: a systematic review. Acad Emerg Med 2018;25:552-65.

4. Scaggs TR, Glass DM, Hutchcraft MG, Weir WB. Prehospital ketamine is a safe and effective treatment for excited delirium in a community hospital based EMS system. Prehosp Disaster Med 2016;31:563-9.

5. Vilke GM, Debard ML, Chan TC, et al. Excited Delirium Syndrome (ExDS): defining based on a review of the literature. J Emerg Med 2012;43:897-905.

6. Campeau AG. The space-control theory of paramedic scenemanagement. Symb Interact 2008;31:285-302.

7. Maguire BJ, O' Meara PF, Brightwell RF, O' Neill BJ, Fitzgerald GJ. Occupational injury risk among Australian paramedics: an analysis of national data. Med J Aust 2014;200:477-80.

8. British Columbia Emergency Health Services. (2019). BCEHS Treatment Guidelines. Available at: https:// handbook.bcehs.cal

9. Hall RI, Sandham D, Cardinal P, et al. Propofol vs midazolam for ICU sedation. Chest 2001;119:1151-9.

10.Pfizer. Product Monograph. Midazolam Injection USP 2020. Available at: www.pfizer.ca/sites/default/ files/201712/2017.10.25_Midazolam_PM_PF_E_210318.pdf

11. Riddell J, Tran A, Bengiamin R, Hendey GW, Armenian $P$. Ketamine as a first-line treatment for severely agitated emergency department patients. Am J Emerg Med 2017;35:1000-4.

12. Chan EW, Taylor DM, Knott JC, et al. Intravenous droperidol or olanzapine as an adjunct to midazolam for the acutely agitated patient: a multicenter, randomized, double-blind, placebo-controlled clinical trial. Ann Emerg Med 2013;61:72.

13.Knott JC, Taylor DM, Castle DJ. Randomized clinical trial comparing intravenous midazolam and droperidol for sedation of the acutely agitated patient in the emergency department. ibid. 2006;47:61-7.

14. Isenberg D, Jacobs D. Prehospital agitation and sedation trial (PhAST): a randomized control trial of intramuscular haloperidol versus intramuscular midazolam for the sedation of the agitated or violent patient in the prehospital environment. Prehosp Disaster Med 2015;30:491-5.

15. Horton C. Prehospital use of ketamine for agitated patients. NEJM Journal Watch Emergency Medicine 2014. Available at: www.jwatch.org/na36508/2014/12/16/prehospital-useketamine-agitated-patients

16.Scheppke KA, Braghiroli J, Shalaby M, Chait R. Prehospital use of IM ketamine for sedation of violent and agitated patients. West J Emerg Med 2014;15:736-41.

17. Olives T, Nystrom P, Cole J, Dodd K, Ho J. Intubation of profoundly agitated patients treated with prehospital ketamine. Prehosp Disaster Med 2016;31:593-602.

18. Sibley A, Mackenzie M, Bawden J, et al. A prospective review of the use of ketamine to facilitate endotracheal intubation in the helicopter emergency medical services (HEMS) setting. Emerg Med J 2011;28:521.

19.Linder LM, Ross CA, Weant KA. Ketamine for the acute management of excited delirium and agitation in the prehospital setting. Pharmacotherapy 2018;38:138-51.

20.McKay WP. Intravenous analgesia for out-of-hospital traumatic pain in adults: Ketamine gives a greater reduction in pain than morphine but causes more adverse effects. Evid Based Nurs 2013;16:58.

21.Andolfatto G, Innes K, Dick W, et al. Prehospital analgesia with intranasal ketamine (PAIN-K): a randomized doubleblind trial in adults. Ann Emerg Med 2019;74:241-50.

22. Buckland D, Crowe R, Cash R, et al. Ketamine in the prehospital environment: a national survey of paramedics in the United States. Prehosp Disaster Med 2018;33:23-8.

23. Mankowitz SL, Regenberg P, Kaldan J, Cole JB. Ketamine for rapid sedation of agitated patients in the prehospital and emergency separtment settings: a systematic review and proportional meta-analysis. J Emerg Med 2018;55:670-81.

24.Gangathimmaiah V, Le Cong M, Wilson M, et al. Ketamine sedation for patients with acute behavioral disturbance during aeromedical retrieval: a retrospective chart review. Air Med J 2017;36:311-4.

25. Hollis GJ, Keene TM, Ardlie RM, Caldicott DG, Stapleton SG. Prehospital ketamine use by paramedics in the Australian Capital Territory: a 12 month retrospective analysis. Emerg Med Australas 2017;29:89-95.

26. New South Wales Ambulance Service. NSWAS Protocol and Pharmacology Sydney, Australia 2019. Available at: https:// nswambulance.wordpress.com/

27. Ambulance Victoria. Clinical Practice Guidelines 2020. Available at: www.ambulance.vic.gov.au/paramedics/clinicalpractice-guidelines/ 


\section{References (continued)}

28. Keseg D, Cortez E, Rund D, Caterino J. The use of prehospital ketamine for control of agitation in a metropolitan firefighter-based EMS system. Prehosp Emerg Care 2015;19:110-5.

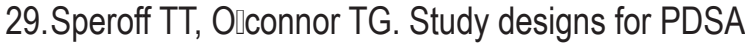
quality improvement research. Qual Manag Health Care 2004;13:17-32.

30.BC Emergency Health Services. (2019). Fact Sheets and FAQs. Available at: www.bcehs.ca/about/accountability/factsheets-and-faqs

31.Sessler CN, Gosnell MS, Grap MJ, et al. The Richmond Agitation-Sedation Scale. Am J Respir Crit Care Med 2002;166:1338-44.

32. Kovacs G, Law A. Airway Intervention and Management in
Emergencies (AIME) 2019. Available at: www.aimeairway. $\mathrm{cal}$

33. Williams B, Boyle M, O'Meara P. Can undergraduate paramedic and nursing students accurately estimate patient age and weight? Prehosp Disaster Med 2010;25:171-7.

34.Lieb N, Gluckman WA. Accuracy of adult patient weight estimation by EMS providers. Prehosp Emerg Care 2004;8:96-7.

35.Martin DR, Soria DM, Brown CG, et al. Agreement between paramedic-estimated weights and subsequent hospital measurements in adults with out-of-hospital cardiac arrest. Prehosp Disaster Med 1994;9:54.

36. Burnett AM, Salzman JG, Griffith KR, Kroeger B, Frascone RJ. The emergency department experience with prehospital ketamine: a case Series of 13 patients. Prehosp Emerg Care 2012;16:553-9. 